\title{
A POSITIONING FREE CALIBRATION METHOD FOR MOBILE LASER SCANNING APPLICATIONS
}

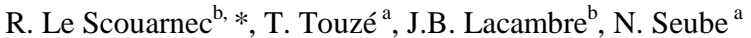

${ }^{a}$ ENSTA Bretagne, Dept STIC/OSM, Brest - (nicolas.seube, thomas.touzé)@ensta-bretagne.fr

b iXBlue, Marly-le-roi, (romain.le-scouarnec, jean-baptiste.lacambre)@ixblue.com

KEY WORDS: Mobile laser scanning, Calibration, Boresight, Positioning-free, Plane features.

\begin{abstract}
:
Mobile laser scanning are likely to find more and more applications for high density 3D environmental data. A mobile laser scanning system is composed by three subsystems: a GNSS (Global Navigation Satellite System) that provides position information, an INS (Inertial Navigation System) for attitude determination, and a LiDAR (Light Detection And Ranging). The accuracy of the geolocated LiDAR returns depends on the accuracy of each instrument but also on the bore-sighting parameters and the lever arms between the instruments. Indeed, an imperfect calibration may lead to systematic errors. Calibration may then become the limiting factor of Terrestrial Laser scanning if it is not tackled seriously. Moreover [Ø], it is important to have a reliable value of the calibration precision. This paper presents a new positioning free procedure for the estimation of the LiDAR bore-sighting parameters. Since this method is static, lever arms do not affect the boresight calibration and positioning is not required. That makes the methodology immune to GPS errors. Finally, since it is based on a rigorous mathematical model, it can provide a reliable boresight quality factor. First, the boresight determination problem is explained and existing calibration procedures are introduced. After having explained their drawbacks, a new procedure that tries to overcome these limitations is described. Tests from simulations and real datasets are also presented to illustrate our approach.
\end{abstract}

\section{INTRODUCTION}

\subsection{The INS-LiDAR system}

A mobile laser scanning system is composed by a LiDAR which gives the distance to the target, an INS that provides the system orientation and a GNSS that estimates the position in a global frame. Combined altogether, these 3 instruments enable the geolocalization of the LiDAR points in a global frame such as the [ECEF] frame (Earth Centered Earth fixed).

A geo-location equation for this system is:

$$
\mathbf{X}_{L i D A R}^{e c e f}=\mathbf{X}_{G P S}^{e c e f}+C_{n}^{e c e f} C_{I N S}^{n}\left(\mathbf{b}^{I N S}+\left(C_{L i D A R}^{I N S}\right) \mathbf{X}_{L i D A R}^{L i D A R}\right)
$$

where

Measurements:

$\mathbf{X}_{\text {LiDAR }}^{\text {LiDAR }}$ : Range measured by the LiDAR in [LiDAR] frame

$\mathbf{X}_{G P S}^{e c e f}$ : GPS antenna position

$C_{I N S}^{n}: \quad$ Rotation matrix calculated thanks to the INS data

$C_{n}^{e c e f}:$ Rotation matrix from [n] to [ECEF]

Parameters to be calibrated:

$C_{L i D A R}^{I N S}$ : Rotation matrix from [LiDAR] frame to [INS] frame

$\mathbf{b}^{I N S}$ : Lever arm between the LiDAR and the GPS antenna

Boresight calibration consists in determining the relative orientation of the LiDAR to the INS described by $C_{L i D A R}^{I N S}$.

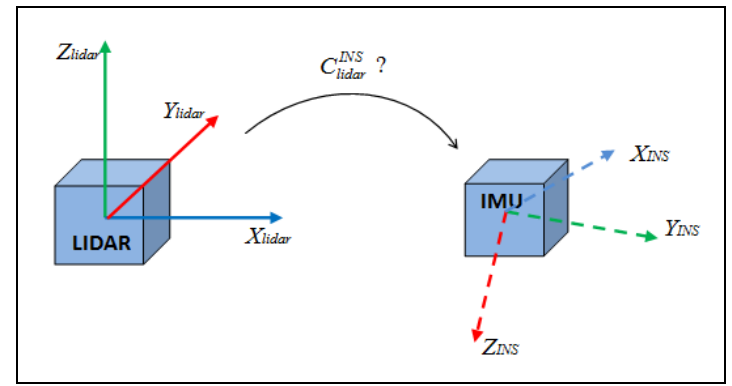

Figure 1: Relative orientation between LiDAR and INS

For terrestrial laser scanning, the error due to the boresight should be inferior to the cm-level in order to be lower to positioning uncertainty, which is less than $1 \mathrm{~cm}$ with INS/GNSS hybridized post-processed data.

At a given range, the shift error $\varepsilon_{\text {boresight }}$ created by a boresight error $\theta_{\text {boresight }}$ can be approximated by this expression:

$$
\varepsilon_{\text {boresight }}[\mathrm{m}]=\operatorname{Range}[\mathrm{m}] \times \theta_{\text {boresight }}
$$

Therefore with a 50 meter range, $\theta_{\text {boresight }}$ needs to be inferior to $10^{-2}$ degree in order to get an error $\varepsilon_{\text {boresight }}$ inferior to $1 \mathrm{~cm}$.

Mechanically, it is difficult to know precisely the relative orientation of the LiDAR to the INS, i.e., $C_{L i D A R}^{I N S}$ is known within about a few degrees. Then, the remaining misalignment needs to be estimated computationally via a calibration procedure. Mathematically, the misalignment can be modeled by a rotation matrix $C_{I N S^{*}}^{I N S}$ called boresight matrix.

Corresponding author. 


$$
C_{L i D A R}^{I N S}=C_{I N S^{*}}^{I N S} C_{L i D A R}^{I N S^{*}}
$$

where $C_{L i D A R}^{I N S}$ : "true" relative orientation $C_{\text {LiDAR }}^{I N S^{*}}$ : Approximate mechanical relative orientation

[INS] can be defined as the "true" INS frame whereas [INS"] is the approximate INS frame resulting from the approximate rotation $C_{L i D A R}^{I N S^{*}}$.

The Boresight matrix $C_{I N S}^{I N S}$ is characterized by the 3 boresight angles. These boresight angles are defined in the figure 2

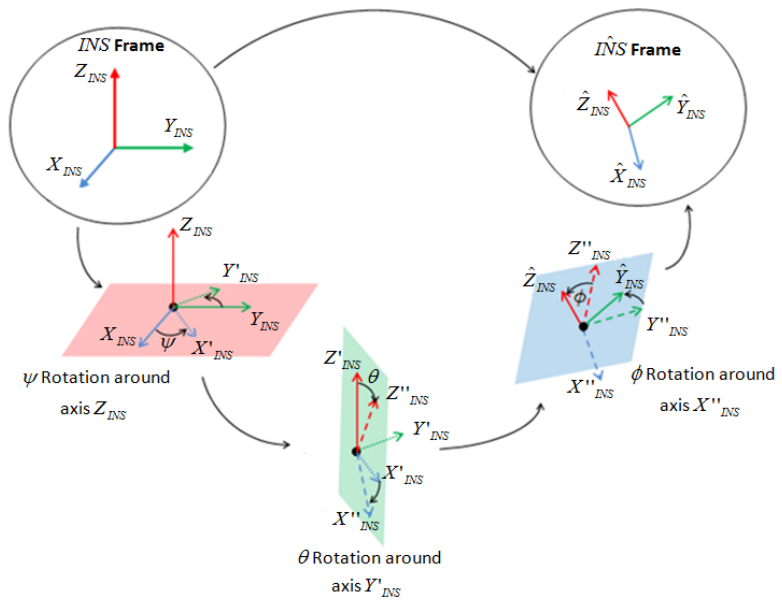

Figure 2: Boresight angles definition

Suitable observations have to be found to correct this misalignment. Two main approaches have been identified in the literature to calibrate the bore-sighting parameters:

- Data driven methods.

- Model driven methods.

These methods are developed in the next paragraph.

\subsection{Previous researches}

Most of the work dedicated to boresight calibration has been done for airborne LiDAR for which it is critical since the distances are superior to 100 meters. Current Terrestrial LiDAR calibrations are inspired by these methods. That is why they are presented hereafter.

\section{Data Driven methods}

Data driven methods do not use raw data but directly use the processed LiDAR points. These methods are probably the most popular because they do not require the access to INS or GPS data. However, they are also the less rigorous. They consist in estimating a rotation matrix $R$ and a translation vector $\mathbf{T}$ that minimize the distance between LiDAR points observed from several strips that partially superimpose. This method implies that an operator selects "target points" that appear in several point clouds so that the algorithm can be run. Despite their simplicity, data driven methods present significant drawbacks:

- The method is not rigorous since $R$ and $\mathbf{T}$ do not model correctly the boresight and lever arm errors (see Eq. 2).

- It is time consuming because common target points have to be identified in the LiDAR point clouds.
- This point selection is user-dependent and so are the performances of the adjustment.

\section{System Driven methods}

Unlike data driven methods, system driven methods rely on the mathematical model described by the geo-location equation (Eq. 1). Hence, the error model associated with the parameters $\Delta \mathbf{b}$ and $C_{I N S^{*}}^{I N S}$ is:

$$
\mathbf{X}_{L i D A R}^{e c e f}=\mathbf{X}_{G P S}^{e c e f}+C_{n}^{e c e f} C_{I N S}^{n}\left((\mathbf{b}+\Delta \mathbf{b})+\left(C_{I N S^{*}}^{I N S} C_{L i D A R}^{I N S^{*}}\right) \mathbf{X}_{L i D A R}^{L i D A R}\right)(\mathbf{2})
$$

where $\quad \Delta \mathbf{b}:$ Bias on the lever arm between LiDAR and GPS. $C_{I N S^{*}}^{I N S}$ : Boresight matrix.

Sometimes this model is more complete and also calibrates a LiDAR range bias.

The first methods developed were similar to data driven methods but use a different error model described by (Eq. 2). Although this model is more accurate, it still has some drawbacks:

- It is sensible to GPS errors

- Users still have to select points which makes it quite long and user-dependent.

In order to improve the calibration process, (Skaloud, 2006) proposed to use features such as plans instead of comparing pair of points.

This method is based on the assumption that LiDAR points belong to a same feature and therefore satisfy the equation modeling the feature. For instance, if the feature is a plan, all points belonging to the plan should satisfy Eq. (3):

$$
n_{1} x+n_{2} y+n_{3} z+n_{4}=0
$$

where $\quad \mathbf{N}^{\text {ecef }}=\left[\begin{array}{lll}n_{1} & n_{2} & n_{3}\end{array}\right]^{T}$ : Normal vector to the plan

$$
\mathbf{X}^{\text {ecef }}=\left[\begin{array}{lll}
x & y & z
\end{array}\right]^{T} \quad: \text { LiDAR return }
$$

This approach has many advantages. First, using a feature model imposes a strong common hypothesis on a cluster of points. Moreover, it is easier to select a cluster of points that belong to a same plan than a pair of "target points". This point selection can even be automated (Skaloud, 2007) by detecting planar surfaces. It proved to be an efficient method for airborne LiDAR. That is why a similar procedure was then adopted for terrestrial LiDAR calibration (Rieger, 2007).

However, the context is different when it comes to terrestrial LiDAR:

- Distances are much shorter. Consequently, it makes the boresight error less observable.

- GPS errors have a bigger impact on small ranges and multipath is more likely to happen in a terrestrial context than in an aerial context.

- It is more difficult to find different plane orientations, which is necessary to achieve a good calibration. Most planes are horizontal or vertical.

- This method proved to be sensible to INS biases 


\section{CALIBRATION LIDAR-INS}

\subsection{Improvements introduced by the proposed method}

Conceptually, the proposed calibration approach is radically different from classical methods. Classical calibration procedures are based on points (positions) whereas the suggested method is based on vectors (orientations).

In practice, the main difference is that each scan has to be carried out statically.

Important consequences ensue from these 2 remarks:

- The method is position-free and GPS errors do not interfere.

- Boresight angles are calculated in the navigation frame [n]. It makes the boresight calibration immune to INS biases.

- Orientation data are less noisy than position data.

\subsection{Presentation of the calibration procedure}

The objective is to estimate the boresight angles $(\phi, \theta, \psi)$ and to ensure reliable standard deviations associated with these angles.

As mentioned in the state of the art, classic methods already use planar features to calibrate the boresight angles using the hypothesis that LiDAR returns belong to a same plan and thus verify the following equation:

$$
\left(\overline{\mathbf{N}}^{\text {ecef }}\right)^{T} \cdot\left(\begin{array}{c}
\mathbf{X}_{G P S}^{\text {ecef }}+C_{n}^{\text {ecef }} C_{I N S}^{n}\left((\mathbf{b}+\Delta \mathbf{b})+\left(C_{I N S^{*}}^{I N S} C_{L i D A R}^{I N *^{*}}\right) \mathbf{X}_{L i D A R}^{L i D A R}\right) \\
1
\end{array}\right)=0
$$

where $\quad\left(\overline{\mathbf{N}}^{\text {ecef }}\right)^{T}=\left[\begin{array}{llll}n_{1} & n_{2} & n_{3} & n_{4}\end{array}\right]=\left[\begin{array}{ll}\mathbf{N} & n_{4}\end{array}\right]$

These methods are run dynamically, i.e., the vehicle is moving as LiDAR return points are acquired.

The proposed procedure also uses planar features but the scanning is performed statically. As shown on Figure 3 LiDAR return points acquired statically are lined up and form a scanline u.

As all scanlines belong to a same plan, their direction vectors are orthogonal to the normal of the plan. (Eq. 4) becomes:

$$
\begin{aligned}
\left\langle\mathbf{N}^{\text {ecef }}, \mathbf{u}^{\text {ecef }}\right\rangle & =0 \\
\left\langle\mathbf{N}^{n}, \mathbf{u}^{n}\right\rangle & =0
\end{aligned}
$$

Since $\mathbf{u}$ and $\mathbf{N}$ are orientations and not positions, there is no longer need for global positioning. That is why the previous equation is also valid is the navigation frame [n].

Using the geo-location equation, the previous equation can be developed as a function of boresight angles:

$$
f=\left(\mathbf{N}^{\mathbf{n}}\right)^{T} \cdot\left(C_{I N S}^{n} C_{I N S^{*}}^{I N S} C_{L i D A R}^{I N S^{*}} \mathbf{u}_{\text {LiDAR }}^{\text {LidAR }}\right)=0
$$

That new equation is position-free since only orientations are involved. Consequently, GPS and lever-arms errors do not appear anymore in the equation. That is a great improvement because GPS measurements are the noisiest data.

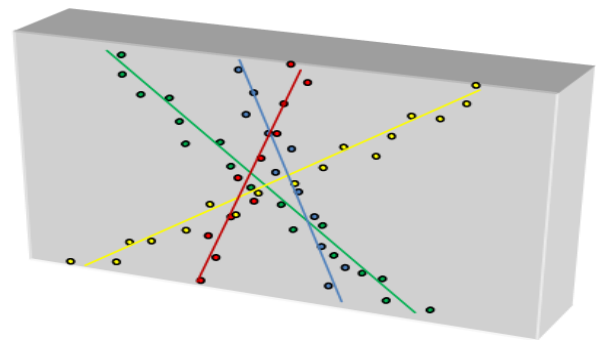

Figure 3: 4 Scanning lines

Mathematical formulation of the positioning-free calibration Parameters and observations:

$$
\mathbf{x}=\left[\begin{array}{c}
\phi \\
\theta \\
\psi \\
\left(\mathbf{N}_{\mathbf{1}}\right)^{n} \\
\vdots \\
\left(\mathbf{N}_{p}\right)^{n}
\end{array}\right]
$$$$
\mathbf{l}_{\mathbf{i}}=\left[\begin{array}{c}
\text { roll }_{i} \\
\text { pitch }_{i} \\
\text { heading }_{i} \\
\mathbf{u}_{\mathbf{i}}^{\text {LiDAR }}
\end{array}\right]
$$

where $\quad \mathbf{x}$ : is the parameter vector. It contains the boresight angles and the normals of the $p$ scanned planes. $\mathbf{l}_{i}:$ is the observation vector related to the $\mathrm{i}^{\text {th }}$ observation expressed in the [LiDAR] frame.

The boresight angles and the plane parameters are simultaneously estimated with a Gauss-Helmert model (also known as Combined Least Squares) with additional constraints on the normals as they are unit vectors.

\section{Gauss-Helmert Model}

$$
A \Delta \mathbf{x}+B \Delta \mathbf{l}+\mathbf{w}_{\mathbf{0}}=\mathbf{0}
$$

Where $\quad \mathbf{A}=\left.\frac{\partial f(\mathbf{x}, \mathbf{l})}{\partial \mathbf{x}}\right|_{\hat{\mathbf{x}}, \hat{\mathbf{I}}}:$ Jacobian matrix of $f$ with respect to $\mathbf{x}$

$$
\begin{aligned}
& \mathbf{B}=\left.\frac{\partial f(\mathbf{x}, \mathbf{l})}{\partial \mathbf{l}}\right|_{\hat{\mathbf{x}}, \mathbf{l}}: \text { Jacobian matrix of } f \text { with respect to } \mathbf{l} \\
& \mathbf{w}_{\mathbf{0}=f(\hat{\mathbf{x}}, \hat{\mathbf{l}}) \quad: \text { Misclosure vector }}
\end{aligned}
$$

The constraints:

Each normal has for constraint to be a unit vector. These constraints also have to be linearized:

$$
\begin{gathered}
g\left(\mathbf{N}_{\mathbf{i}}\right)=\mathbf{N}_{\mathbf{i}}^{T} \mathbf{N}_{\mathbf{i}}-1=0 \\
C \Delta \mathbf{\Delta x}+w_{c}=0
\end{gathered}
$$

Where $\quad C=\left.\frac{\partial g(\mathbf{x}, \mathbf{l})}{\partial \mathbf{x}}\right|_{\hat{\mathbf{x}}, \hat{\mathbf{l}}}:$ Jacobian matrix of $g$ with respect to $\mathbf{x}$ 


\section{Solution:}

The Gauss-Helmert model with constraints can be solved iteratively using Lagrange multipliers. It leads to a normal equation which is linear and that can easily be solved:

$$
\begin{gathered}
H \mathbf{\Delta} \mathbf{X}+\mathbf{w}=0 \\
\left\{\begin{array}{l}
H=A^{T} W A+C^{T} W_{c} C \\
\mathbf{w}=A^{T} W \mathbf{w}_{\mathbf{0}}+C^{T} W_{c} \mathbf{w}_{\mathbf{c}}
\end{array} \quad \text { with } W=\left(B W_{l}^{-1} B^{T}\right)^{-1}\right.
\end{gathered}
$$

Where : $W_{l}=\Sigma_{l}^{-1}$ : Weight matrix of the observations

$$
W_{c}=\Sigma_{c}^{-1} \quad \text { : Weight matrix of the constraints }
$$

The variances of the constraints have to be much inferior to measurements variances. Thus, the constraints are equivalent to a penalty method.

Hence, at the $\mathrm{k}^{\text {th }}$ iteration, the parameter vector is updated recursively until a defined convergence criterion is met:

$$
\begin{gathered}
\mathbf{X}_{\mathbf{k}}=\mathbf{X}_{\mathbf{k}-\mathbf{1}}+\Delta \mathbf{X}_{k}=\mathbf{X}_{\mathbf{k}-\mathbf{1}}+H^{-1}\left(-\mathbf{w}_{\mathbf{0}}\right) \\
\Sigma_{x x}=\sigma^{2} H^{-1}
\end{gathered}
$$

Where $\sigma^{2}$ : unit variance factor

The least squares solution is useful because it provides standard deviations for the estimated parameters. Then the user can refer to these values to evaluate its calibration precision:

$$
\left[\left(\sigma_{\phi} \sigma_{\theta} \sigma_{\psi}\right) \boldsymbol{\sigma}_{\mathbf{N} \mathbf{1}} \cdots \boldsymbol{\sigma}_{\mathbf{N p}}\right]=\sqrt{\operatorname{diag}\left(\Sigma_{x x}\right)}
$$

The validity of the standard deviations is presented in part 3 .

\section{In practice:}

The algorithm inputs are:

- LiDAR returns coordinates $\left\{\mathbf{X}_{\text {LiDAR }}^{L i D R}\right\}$

- $\quad$ INS orientations $\{($ roll,pitch,heading $)\}$

- $\quad$ The approximate LiDAR-INS configuration $C_{L i D A R}^{I N S^{*}}$.

Transforming the INS orientations in a rotation matrix $C_{I N S}^{n}$ is straightforward.

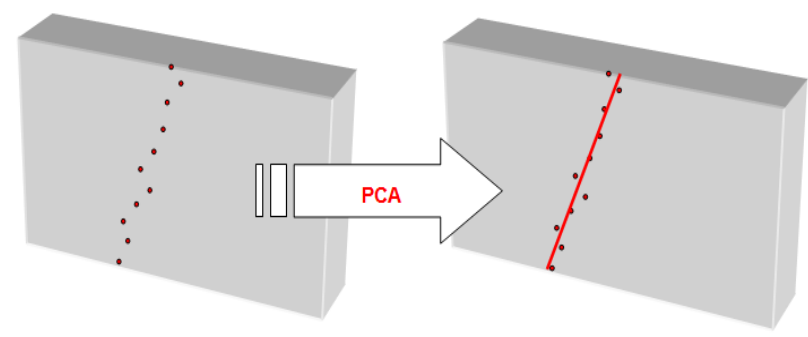

Figure 4: Scanning line estimation
On the other hand, the LiDAR returns cannot be used directly. The scanning lines need to be computed. A Principal Component Analysis (PCA) is carried out to estimate the direction vector $\mathbf{u}_{\mathbf{i}}^{\text {LiDAR }}$ of the $i^{\text {th }}$ scanline in the [LiDAR] frame as shown on figure 4 .

Moreover, the normal vectors need to be approximated in order to accelerate the convergence of the algorithm. They are initialized by the cross product of two randomly chosen direction vectors $\hat{\mathbf{u}}_{\mathbf{i}}{ }^{n}$ and $\hat{\mathbf{u}}_{\mathbf{j}}{ }^{n}$. If these 2 direction vectors belong to the same plan and are not collinear, we have:

$$
\hat{\mathbf{N}}^{n}=\hat{\mathbf{u}}_{\mathbf{i}}{ }^{n} \times \hat{\mathbf{u}}_{\mathbf{j}}{ }^{n}
$$

where

$$
\begin{aligned}
& \hat{\mathbf{u}}_{\mathbf{i}}{ }^{n}=C_{I N S}^{n} C_{\text {LiDAR }}^{I N S^{*} \mathbf{u}_{\mathbf{i}}{ }^{\text {lidar }}} \\
& \hat{\mathbf{u}}_{\mathbf{i}}{ }^{n}=C_{I N S}^{n} C_{\text {LiDAR }}^{I N S^{*}} \mathbf{u}_{\mathbf{j}}{ }^{\text {lidar }}
\end{aligned}
$$

Note that the boresight matrix does not appear in the expressions of $\hat{\mathbf{u}}_{\mathbf{i}}{ }^{n}$ and $\hat{\mathbf{u}}_{j}{ }^{n}$ since boresight angles have not been estimated yet. $\hat{\mathbf{N}}^{n}$ is just an approximate initialization.

\section{RESULTS}

\subsection{Experimental setups}

\section{System configuration}

The instruments used are a LiDAR Leica HDS6200 which has a precision lower than $5 \mathrm{~mm}$ for ranges inferior to $25 \mathrm{~m}$ and lower than to $1 \mathrm{~cm}$ for ranges inferior to $50 \mathrm{~m}$. The INS is a iXBLUE Landins which provides the attitude with a $0.02^{\circ}$ accuracy for the roll and pitch and a $0.1^{\circ}$ accuracy for the heading.

The LiDAR system configuration is shown on Figure 5.

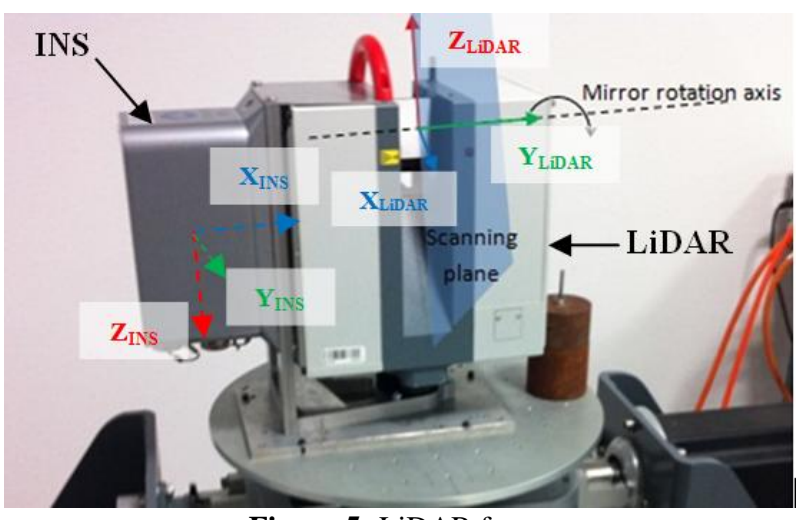

Figure 5: LiDAR frame

\section{Definition of the orientations}

For simulations and real tests presented in this paper, only 2 plans were used: one vertical and one horizontal (typically the floor or the ceiling). This choice is motivated by practical considerations: most walls scanned in terrestrial laser scanning are horizontal or vertical plans.

The orientation choices are important for a successful calibration. One can show the following scanning orientations are relevant with this system configuration (the demonstration is beyond the scope of this paper): 
- Scanning the vertical plan with $\alpha$ angles close to $0^{\circ} / 180^{\circ}$. Scanning the vertical plan with $\alpha$ angles close to $\pm 70^{\circ}$.

- Scanning the horizontal plan with the LiDAR system tilted with $\beta$ angles superior to $20^{\circ}$ in absolute value.

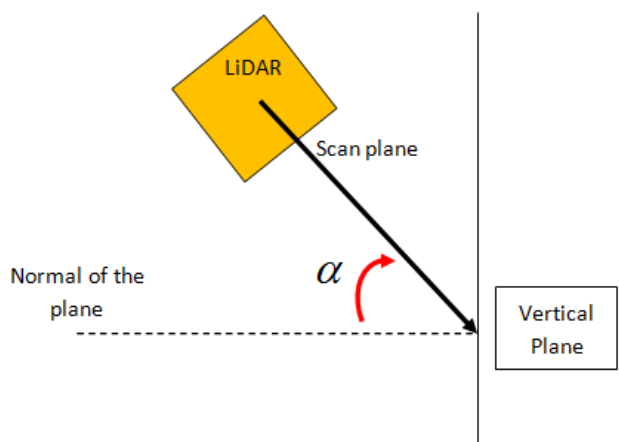

Figure 6: Vertical plan scanned with an $\alpha$ angle (view from above)

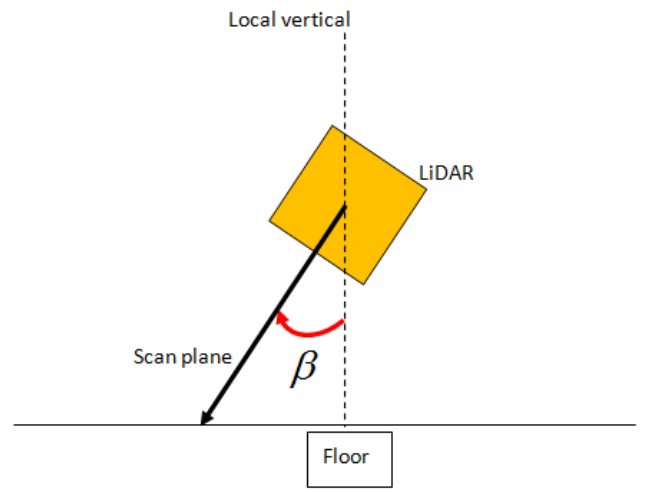

Figure 7: Horizontal plan (floor) scanned with a $\beta$ angle

\subsection{Simulation results}

The simulator reproduces the configuration presented in the paragraph dedicated to the system configuration. It is used to generate:

- Random boresight angles.

- $\quad p$ plans with specified orientations. They are chosen roughly vertical or horizontal.

- Noisy INS orientations data (bias and noise) with specified orientations.

- Noisy LiDAR data.

The results presented hereafter are obtained with 2 plans. The $1^{\text {st }}$ plan is approximately vertical $\left(\right.$ at $\left.\pm 5^{\circ}\right)$ and the $2^{\text {nd }}$ plan is almost horizontal (at $\pm 5^{\circ}$ ). Each wall is scanned with a given sequence of orientations.

The error distributions are displayed in Figure 9. For the 3 angles, the standard deviation given in Table 8 are inferior to $10^{-2}$ degree which was the objective given in the introduction.

\begin{tabular}{|c|c|c|}
\hline$\sigma_{\phi}$ & $\sigma_{\theta}$ & $\sigma_{\psi}$ \\
\hline $0.001^{\circ}$ & $0.0027^{\circ}$ & $0.0004^{\circ}$ \\
\hline
\end{tabular}

Table 8: Standard deviations of the boresight angles estimated with the simulated data
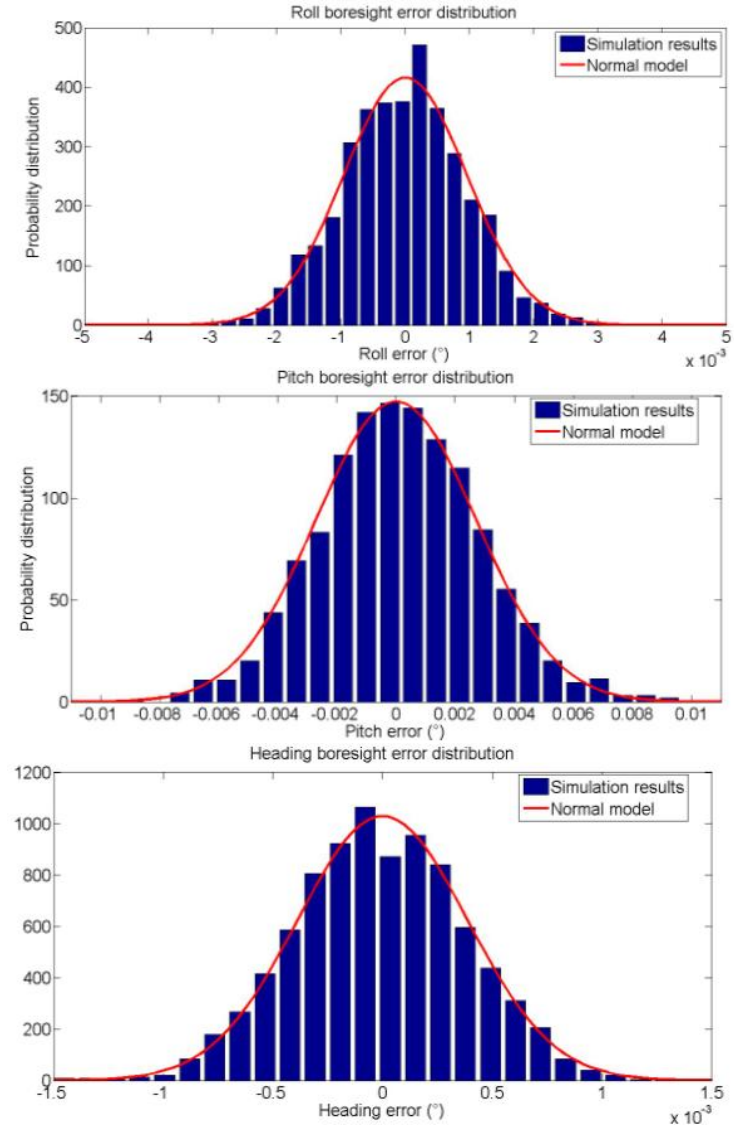

Figure 9: Distribution of the boresight errors (roll/pitch/heading)
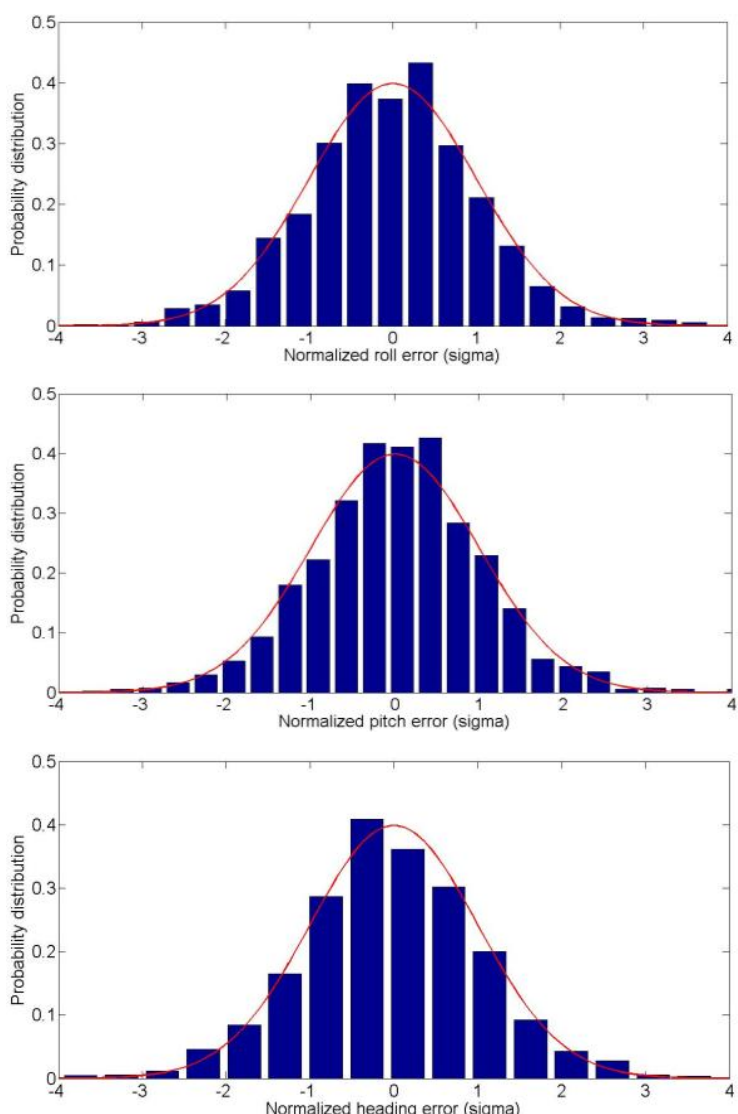

Figure 10: Roll/Pitch/Heading normalized residuals 
To validate the standard deviations $\hat{\sigma}_{\phi}, \hat{\sigma}_{\theta}, \hat{\sigma}_{\psi}$ estimated with this procedure, the normalized residuals are calculated.

$$
\varepsilon_{n o r m, \phi}=\frac{\phi-\hat{\phi}}{\hat{\sigma}_{\phi}}, \varepsilon_{n o r m, \theta}=\frac{\theta-\hat{\theta}}{\hat{\sigma}_{\theta}}, \varepsilon_{\text {norm }, \phi}=\frac{\phi-\hat{\phi}}{\hat{\sigma}_{\phi}}
$$

Where $\phi, \theta, \psi:$ generated boresight angles

$$
\hat{\phi}, \hat{\theta}, \hat{\psi} \text { : estimated boresight angles }
$$$$
\varepsilon_{\text {norm }, \phi}, \varepsilon_{\text {norm }, \theta}, \varepsilon_{\text {norm }, \psi}: \text { normalized residuals }
$$

If the standard deviations are well estimated, then the normal residuals should be distributed according to a standard normal distribution. That means that most normalized residuals (99.7\%) should be between -3 and $+3 \quad\left(\sigma_{\text {norm }}=1\right)$. The normalized residuals distributions are shown on Figure $\mathbf{1 0}$ and prove that the estimated standard deviations are relevant.

\subsection{Experimental results}

The calibration can be performed on a vehicle, but also directly at the laboratory on a rotating table.

Tests were run in indoor with 2 plans scanned simultaneously: a vertical wall and the floor. The LiDAR scanlines were extracted automatically with a RANSAC algorithm.

The calibration procedure was run several times and estimated parameters are compared in Table 11 and Table 12.

\begin{tabular}{|c|c|c|}
\hline & Boresight angles & Standard deviations \\
\hline Roll & $0.0588^{\circ}$ & $0.0104^{\circ}$ \\
\hline Pitch & $-0.0076^{\circ}$ & $0.0305^{\circ}$ \\
\hline Heading & $-0.2754^{\circ}$ & $0.0323^{\circ}$ \\
\hline
\end{tabular}

Table 11: Results from Test \#1

\begin{tabular}{|c|c|c|}
\hline & Boresight angles & Standard deviations \\
\hline Roll & $0.0469^{\circ}$ & $0.0098^{\circ}$ \\
\hline Pitch & $-0.0055^{\circ}$ & $0.0233^{\circ}$ \\
\hline Heading & $-0.2965^{\circ}$ & $0.0304^{\circ}$ \\
\hline
\end{tabular}

Figure 12: Results from Test \#2

Results from Test\#1 and Test\#2 show that the differences are coherent with the estimated standard deviations. However, the performances are not as good as the simulation results.

The precision of calibration depends on 2 criteria: the orientation choices and the precision of the scanline vectors $\mathbf{u}$. Since simulations and real data had roughly the same orientations, the performance differences are explained by the $\mathbf{u}$ vectors.

Real data analyses showed that the plans used could have flaws: they are not as ideally planar as simulation plan can be. The figure 13 illustrates this imperfect planarity.

Second, the scanlines in the simulator are longer than in the real acquisition. Yet, one can easily understand that the signal to noise ratio decreases as the line becomes longer and $\mathbf{u}$ estimation becomes more precise. Additional tests showed that it has a great impact.

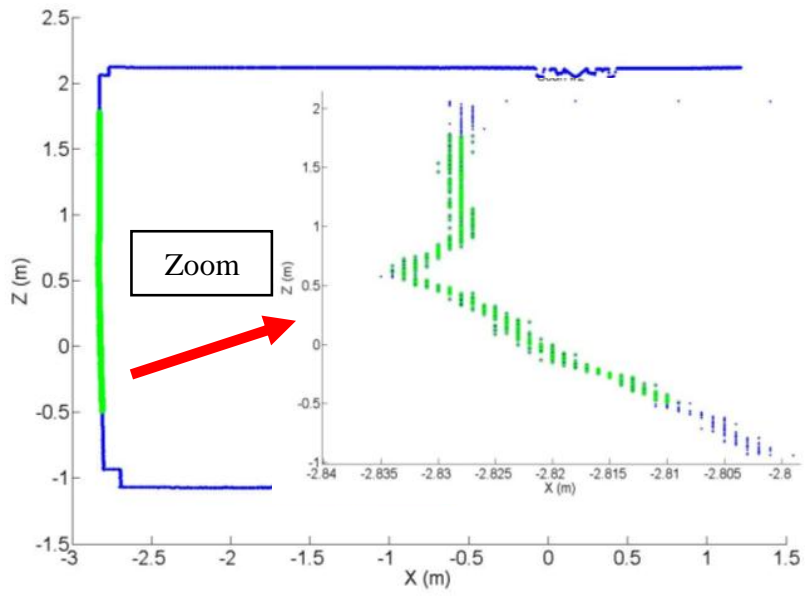

Figure 13: Wall imperfect planarity

Therefore, the $\mathbf{u}$ vector estimation is the main limitation for this calibration procedure. Before starting a calibration, one should scan entirely the plans considered for the calibration to ensure that they are flat enough. A solution to overcome this limitation would be to use normals instead of scanline vectors because normals estimations are less sensible to the wall local imperfections. These static scans would be performed with a laser settled on a rotating platform.

\section{CONCLUSION}

In this paper, the boresight calibration problem was tackled with a new approach: a position-free calibration procedure based on orientations. Consequently, calibration does not depend on GPS precision anymore and can even be run directly in the laboratory before setting up the LiDAR system on the vehicle. Simulations proved the efficiency of the method and real datasets confirmed the coherence of the standard deviations.

The main limitation of this calibration is the estimation of the $\mathbf{u}$ vector. Scanning plans and using their normal is a solution that will be considered in future works.

\section{ACKNOWLEDGEMENTS}

This research project is supported by a grant from the Délégation Générale pour l'Armement (DGA).

\section{REFERENCES}

Skaloud, J., Litchi, D., 2006, Rigorous approach to boresight self-calibration in airborne laser scanning, ISPRS, Journal of Photogrammetry and Remote Sensing, Vol. 61, Issue 6, pp 414415

Skaloud, J.; Schaer, P. Towards automated LiDAR boresight self-calibration. Proceedings of 5th International Symposium on Mobile Mapping Technology, Padova, Italy (29-31 May 2007)

Rieger, P., Studnicka, N., Pfennigbauer, M., Zach, G., 2008. Boresight alignment method for mobile laser scanning systems. Journal of Applied Geodesy. Volume 4, Issue 1, Pages 13-21

Leica HDS6200 datasheet.

http://hds.leica-geosystems.com/en/Leica-HDS6200_64228.htm

iXBlue Landins datasheet.

http://www.ixsea.com/pdf/landins-2011-01-web.pdf 